UDC 81`271; 81`33

DOI: $10.17223 / 24109266 / 13 / 7$

\title{
FEATURES OF DIGITAL WRITING AS A LANGUAGE ACTIVITY IN THE CONTEXT OF MODERN ONLINE COMMUNICATION
}

\author{
E.N. Vorotilina, V.A. Dmitrieva
}

\begin{abstract}
The article focuses on digital writing, which intrudes on the domain of "live" interaction, largely replacing it and manifestating its specific language features. Modern online communication depends on many factors: the accessibility of the media platforms; forms of written online communication (official / unofficial); type of communication. Everyday digital written communication based on Internet channels leads to the fact that not only the conditions of written speech production are changed, but also the perception of the written text, namely, both writing and reading skills alter. With the advent of novel means of interaction such as the Internet, there has been a lot of research and widespread discussion of how digital media influence the change in the written form of communication. The article summarises the features observed on lexical, syntactic, punctuation and graphic levels.

Keywords: digital communication; online communication; Internet; restriction of access to information; telecommunication technologies; written / spoken form of communication; linguistic economy; abbreviation; apocope.
\end{abstract}

\section{Introduction}

Communication is relocating more and more to the Internet. New technologies are emerging that certainly affect the way people communicate. The Internet itself, on the one hand, is the result of the rapid development of science and technology, and on the other hand, it forms the basis and gives varied opportunities for communication in social networks. The latter allows users to independently generate messages and distribute them in the public domain of the Internet. Such a development of telecommunication networks leads to changes in writing skills, which is expressed primarily in the use of certain language means.

Marshall McLuhan's famous aphorism "the medium is the message" from his 1964 book "Understanding Media: The Extensions of Man" [1] proves true in the Internet era when the fusion of the norms of written and spoken language leads to the appearance of an intermediate form- so called electronic discourse or electronic language. Already back in the $60 \mathrm{~s} \mathrm{McLu-}$ han proposed that a medium itself, not the content, should be the focus of study. Nowadays the so-called digital or electronic form of language is under close observation of linguists. The research in the sphere of the electronic language has a long history, with one of the most profound being thebook by David Crystal "Language and the Internet" - the author's attempt to create a new linguistic discipline - "Internet linguistics" [2: 20-23]. 
In everyday digital communication, the manifestations of features of spokenlanguage are frequent, especially, when communication takes place in the form of dialogue and communicators are familiar with each other. Nevertheless, no matter how close the features of this discourse are to spoken language, this does not change the fact that communication is carried outin writing. Therefore, it would be wrong to argue that the boundaries between the spoken and written forms of a language on the Internet are completely blurred. At the same time, there are, of course, noticeable transitions from oral to written, especially at the stylistic level. Texts that should show signs of official style often retain on the Internet the expressions inherent in the author's oral speech (as any handwritten text by one person would retain the features of their handwriting). As a result, this mixture of spoken and written features in digital writing gave birth to a new term in the Russian language, which sounds like oxymoron, - "written-spoken speech", or written-spoken activity" or "written colloquial speech" [3:7-9].

\section{Methods}

The research on the functioning of the language in a written online communication requires the use of the following methods: observation as the main empirical method of studying language phenomena; linguostylistic analysis of the facts of the language at its different levels; elements of semantic analysis and pragmalinguistic analysis; partial sampling method.

\section{Main part}

\section{A few distinguishing feathers of digital writing}

The fundamental difference between communication mediated by modern digital technology from live interpersonal spoken communications is the limited character of the first one compared to the latter. In telephone conversation there is no opportunity for the speaker to get an impression of a non-acoustic sensual expression on the part of the addressee. The same can be said about the video conferences on Skype. The perception of the communication partners covers only what is currently being broadcast on the screen or through loudspeakers. Similarly, written computer-mediated communication has some peculiarities summarised below.

1. Limited character. Digitally-mediated written communication (e.g. SMS) is even more limited. Firstly, in such communication there is no faceto-face contact between the sender and the recipient, i.e., at the time of sending / receiving a message, it is impossible to evaluate how the sender/addressee interprets the message. Secondly, there is no way to hear voice modulations, intonation, laughter, etc., that is, there are not enough addition- 
al means of expression that specify a certain tonality of the conversation. All of the above, however, does not contradict the basic tenets of the science of writing, graphemics (or grammatology in Ignace Gelb's terminology): "Written culture is a collection of texts formed within this form of written linguistic activity" [4: 41]. Online communication has become this form of written-spoken activity ofmodern time.

2. Changes in writing / reading skills. Digital writing covers the most part of modern communication - interpersonal, business, commerce, politics, including academic interaction, replacing "live" contacts in many situations. Instead of discussing a scientific issue on the sidelines with colleagues, an email is preferably sent. Instead of making an appointment by phone, a message on WhatsApp or Viber, etcis sent. Instead of congratulating someone on the festive occasion or, defending a dissertation in person, people send instant messages through SMS. Such digitalisation of everyday communication leads to the fact that not only the conditions of written speech production are changed, but also the perception of the written text. As a result, both writing and reading skills are altering.

3. Levels of intimacy. Another important factor that makes certain changes to the traditionally understood written communication on the Internet is the border between public and personal sphere of communicators. Upon closer examination, we can point out two aspects relevant to the choice of language tools: access to communication and choice of topics. The latter is important to us since it shows the character of relations between communication partners. People close to each other used to write about personal matters, which was not typical of communicators connected only by business or job relations. Nowadays, the Internet brings to the public eye the topics of the personal sphere, which previously could only be discussed in a narrow family circle or with friends. We become the witnesses of socialization of the personal sphere. The reason for this is the individual's need for social resonance in response to their practice of cognition of the world. Private life in the digital age seems to be imbued with the need for social contacts, the desire for interpersonal communication, the desire to be "seen" and "heard", which is consistent with the possibilities of modern development of telecommunication technologies (Twitter, Facebook, VKontakte, Instagram).

4. Levels of accessibility. Opportunities for communication on the Internet can be ranked according to availability into several levels: 1) platforms with unlimited access; 2) platforms with partial restriction of access to information, for example, those conceived for a certain group of communicants; 3) platforms to which a strictly limited number of persons have access (for example, WhatsApp).

On publicly available platforms, the focus remains on expressing one's own opinion on a particular issue, reporting on personal events and experiences, and disseminating maxims and aphoristic statements. At the 
same time, we should remember that any texts in this kind of online communication, be they "tweets" or reply comments to the primary text, are addressed to the public in general, i.e. they do not have a specific person as the addressee.

At the same time, it is necessary to keep in focus the fact that any comment can form the basis for an online dialogue between specific (two or more) participants of communication. It is a frequent practice that website administrators can graphically highlight the comment that has sparked the most lively discussion. But it is not accepted to expect from the publication of the comment the response of specific persons.

Platforms with unlimited access, nonetheless, do have some tools to restrict the circle of participants or "friends", to ban a person who violates norms of behavior, there are settings to limit access to some data, audio or visual materials.

As for the availability of communication of the third level, i.e. platforms with limited access, they include such forms of digital written communication as SMS, E-mail, WhatsApp, Viber and chats on the Internet. The Facebook platform itself, like Twitter and VKontakte, has options for restricting access to communication, so they can be attributed to the second level, i.e. partially accessible for communication platforms. Unlike all sorts of blogs, forums and comments to publications on the Internet, they are unlimitedly available for communication.

5. Anonymity. On some platforms communication is carried out anonymously. This is true in the first place about chats and forums. Chatting dates back to the $1980 \mathrm{~s}$, but this type of written communication became popular in the 1990s due to young people who enjoyed spending their free time chatting ("chatitsa" as the borrowing sounds in Russian). Not only the younger generation uses the chat, it now functions as an attachment to television and radio programs, the target audience of which is mainly represented by the older generations. Chats based on the results of various television and radio programs invite the audience to discuss problems together with experts in the relevant field. Forums are usually topic oriented and anybody interested in the subject under discussion can join the chart staying actually anonymous. The names under which participants of chats and forums come online are rarely their real names. Even if the policy of the cite demands the real personal name and data, it can be easily made up by the user. Another area of application of written communication in the form of a chat is the so-called "hot lines" where one can get advice on pressing vital issues online. The anonymity of the chat plays an important role.

\section{Language constituents of digital writing in online communication}

Researchers use a variety of terms referring to this newly formed synthesis of written and spoken language: 'Computer Mediated Communica- 
tion' (Herring, 1996), 'electronic language' (Collot \& Belmore, 1996), 'electronic discourse' (Davis \& Brewer, 1997; Panckhurst, 2006), 'interactive written discourse' (Werry, 1996), 'Netlish', 'Weblish', 'Internet language', 'cyberspeak', 'netling' (Thurlow, 2001), 'netspeak' (Thurlow, 2001; Crystal, 2001). 'cyberlanguage'(MacFadyen, Roche, \& Doff, 2004). All these names suggest the idea that a new system of communication has emerged. However, we share the viewpoint that there is no a single concept "Internet language". The terms mentioned above, such as "cyber slang," "network language," or "online language," can only be misleading.

Peter Schlobinski in his work "Fundamental Questions of Linguistics" precedes our further discussion of the specifics of language on the Internet: "The analysis of communication forms on the Internet shows that there are numerous language variations between and within individual communicative practices. The Internet constructs a complex linguistic space, which develops considering various parameters (means of communication, origin of the user, program or platform of communication, written or oral form of communication). Depending on the combination of these parameters, separate functional genres and styles of online communication are formed. "There is no single language of the Internet, but rather only specific linguistic phenomena that appear with varying degree of frequency" [5:230].

\section{Stylistic features of online communication}

In the analysis of the written form of online communication first of all we notice all kinds of abbreviations / truncations of language units. The economy of language means is observed both at the lexical level (words, phraseological units) and at the syntactic level (phrases and sentences). There are two types of truncations:

1) abbreviations that have their own well-established sound and function as one sounding word (SMS - [esemeska]; OK - [okey]);

2) abbreviations that are realized as such in writing, but when pronounced are fully structural in nature (e.g., - for example).

Both types combine the possibility of saving written characters, which reduces writing efforts. Abbreviations are typical of communication through texting. At one time, the so-called SMS-Newspeak, or "hype of cuts" arose and was very popular in the Russian language. In Russia the transmission of complex reduced information was practiced through such communication device as a pager.

In the German online communication, acronyms are found in online communication (not only in SMS, but also in emails), usually at the end of a written message or in questions (LG - LiebeGrüße; hdl-habdichlieb; gg geht'sgut?; wm(d) - wasmachst (du)?). The above examples demonstrate a letter abbreviation: in order to save language effort, only the initial letters of the words included in the sentence are used. 
At the punctuation level, the absence of punctuation marks is noticeable, which is explained by the frequency of such types of interrogative sentences in the practice of SMS communication and the reluctance to waste efforts to switch from one keyboard register to another.

It is interesting to note that abbreviations borrowed from the English language: lol (laughingoutloud), bb (byebye), ily (I loveyou) become international loanwords, in our case, for German and Russian languages.

The presented illustrations indicate that phrases or whole sentences are subject to reduction more often than individual words, As a rule, the writing addressee resorts to other tactics in the latter case: in German and English, this is an apocope of all syllables after the first one (German: Schwe - Schwester; Mo - Montag; English: bro - brother) or syncope, also called "skeletonization" (German : vlcht - vielleicht; english: sry - sorry; thx - thanks) Users seem to feel comfortable with deciphering such vowelless items as TXT ['text'] and XLNT ['excellent'])Skeletonization is akin to abjad, a consonant alphabet where each character (glyph) stands for a consonant. Such a system is used in Arabic and Hebrew.

Another option for saving effort in written messages is hybridization, i.e. the use of numbers for a written word that have the same sound as the word or part of the word. The meaning of the figure itself is neglected. This abbreviation tactic is based on the principle of rebuses, for example: German: 2fel - Zweifel; gute n8 - GuteNacht !; English: 4 you - foryou.

It would be a mistake to believe that such reductions occur only for the sake of saving effort and due to lack of space for writing. One can recall the art of rewriting ancient manuscripts, which, in addition to the aesthetic, cultural, historical and educational aspects, included the art of saving writing materials: parchment was extremely expensive. In the era of digitalization, there is no concern about the problem of saving material for writing. Digital storage media allow users both to quickly write text, and, if necessary, correct or supplement it, or completely delete it. Abbreviations are woven into the fabric of modern digital writing, which violates the written language norm andfavours oral form. It is not accidental that online communication is often called aspoken-written variety of language. Reduction of all types apocopation, syncopation, contamination was inherent in the oral speech norm before the digital age, but with the advent of online written communication the oral norm rushed into written messages. Examples of this kind of reduction are the forms: ne (instead of eine), is (instead of ist), nich (instead of nicht), hab (instead of habe), sehn (instead of sehen), müssn (instead of müssen), grade (instead of gerade), wars (instead of wares), nochn (instead of nocheinen), aufm (instead of aufdem), haste (instead of hastdu). The proportion of such forms in the texts of written online messages varies depending on the communication format - official or informal nature of communication. In the texts of business online correspondence or monologically or- 
ganized texts of blogs addressed to the general public, the units marked by the verbal $\backslash$ oral $/ s p e e c h$ norm are practically absent. In contrast to the written dialogue of two well-known communicators in WhatsApp, whose messages are saturated with units expressing emotions (interjections: Aaah, jipiii), marked dialectically (moin, tach, gell), containing sociolectic elements (from the language of German youth: chillig, beef) and modal words (dann, halt, ja, wohl).

Under these circumstances, the acronyms in written online communication are not getting fewer. There are two reasons for this: 1) in the context of a dialogue one often needs to quickly respond to a sent message (here it is worth talking about saving time); 2) abbreviated forms have turned into specific stylistic means of written online communication (the addressee proves themselves to be a competent user of new forms of communication). A vivid manifestation of this stylistic tool has become the song MfG written and performed by the German hip-hop group "DasphantastischeVier", the text of which consists entirely of letter abbreviations.

Another characteristic feature of German online communication at the lexical level is the use of borrowings from the English language, the proportion of which according to various studies is, nevertheless, not as high as it might seem at first glance - 1-3\% [6: 9]. Anglicisms are words from the English language that are fully or partially assimilated by the German language system, the words that display the characteristic features of the German grammatical structure and thereby differ from the English words themselves: for example, in the realm of the noun, this is capitalization and identification by grammatical gender (computer $\rightarrow$ derComputer; e-mail $\rightarrow$ dieE-Mail); in the field of the verb - integration into the conjugation system of the German verb (totweet $\rightarrow$ tweetern; toswitch $\rightarrow$ switchen).

The possibility to quickly change the Latin / Cyrillic registers contributes to the frequent appearance of inserts from other languages in the texts of online messages. As for the German language, there is no need to change the register, and foreign-language inserts freely penetrate the corpus of the German language. The reason for that can be a variety of factors: from the desire of the addressee to identify with a certain group and to demonstrate equal knowledge of two languages, for example, among bilinguals, to the need to express irony.

At the syntactic level, in addition to the types of abbreviations already mentioned, there exist a specifically syntactic way to truncate the complete sentence structure - an ellipsis (for example, WasmachstduWochenende?). An elliptic construction is understood as a construction where some grammatically obligatory parts of the sentence are missing, which does not interfere with the understanding of the meaning of this sentence, while the missing elements can be recreated from the context. Therefore, we are not talking about any communicative incompleteness. 
Elliptic reduction of the sentence structure in the framework of written communication was considered before the digital age a violation of the language norm. Today, following Hans Zehetmair, such deviations from the norm are still not welcome. H. Zehetmair, Chairman of the German Spelling Council, criticized the practice of sending via SMS or Twitter news messages which do not take into account the rules for constructing a fully-structured German sentence [7].

Online communication manifests its features at the graphic level as well. The current level of development of engineering and technology allows one to express their thoughts and feelings with the help of spelling and typographic tools. The spelling of words with a lowercase letter in Internet and mobile written textsis striking, while the type of text and the form of communication (official / unofficial) do not play any role, but rather indicate an individual writing style. In the context of official online communication, the principle of writing all words with a small letter can be applied sparingly (in case of capitalization of the beginning of a sentence and proper names) or sequentially (in case of writing all words in lowercase) or consistently (in the case of writing all words in lower case).

The principle of neutralizing the opposition "capital / lowercase letter" is especially noticeable in those languages where capitalization with a capital or lowercase letter has a morphographic meaning that is used to differentiate words of one part of speech from another (for example, in German, where all nouns are capitalized). Writing with uppercase or lowercase has another additional meaning. In the routine texts of the official form of online communication, low case is used, while all words written in capital letters are perceived by the recipients of such online messages on an emotional level as a manifestation of an extreme degree of excitement or indignation. The addressee seeks the most expressive form of designating their emotions.

At the graphical level of online communication, there is a tendency which is opposite to saving the means of expressing a message: repetition of the same letter within one word. For example, "Huraaaaaa!", "Yeaaah!". The repeated letters are usually vowels. This type of repetition refers to phonetic repetitions: in writing it is vowels that are more subject to multiple repetitions because it is possible to "drawl" them in oral utterance, which happens in an emotional situation that is expressed at a super-segment level of utterance. Thus, the repetition of letters is intended to imitate the prosodic features of the sounding speech in a written message.

Expressive-emotional function also comes to the fore with the use of several punctuation marks or repetition of one, most often, a question or exclamation mark. In chat texts, commentaries of online publications, dialogically organized written online communication, an exclamation mark often replaces the normative use of a dot (full-stop) that is perceived by online communicators as the end of utterance / break of communication. The writer 
feels the lack of extralinguistic means to clarify their message, therefore, combinations of punctuation marks, or emoticons appear in its place.

The function of emoticons (or smileys) can have a variety of connotations, sometimes quite contradicting: to express positive or negative attitude, to intensify the message or, on the contrary, to "decrease the force of the flame" in the utterance or sometimes just to indicate rapport and the worry of the sender about the recipient's perception of their utterance [2: 37]. Even less time-consuming than inserting emoticons is the use of single letters or punctuation marks in place of words or whole phrases, (like capital " $X$ " standing for "Kiss you", or letter "O" - for "Hugs").

To conclude, the speed of modern online communication and its growing domination in private, social and business spheres leads to noteablechangines in digital writing. On the one hand, there are multiple examples of saving efforts: linguistic - the use of various kinds of abbreviations (at the word-formation and syntactic level: abbreviations, acronyms, ellipses); physical - the use of the same register, the refusal to use the "Shift" button aftercompleting a message. On the other hand, the differentiation of such important areas of communication (innitially intrinsic to the language) as the transmission of information and the expression of emotions also undergo some transformation. The latter is expressed at the graphic level by excessive use (repetition) of letters and certain punctuation marks (colons, hyphens, exclamation and question marks and their combinations), as well as the use of special pictures - emoticons.

\section{Conclusion}

All the features presented above demonstrate the difference of digital or online writing from both oral and written forms of speech and suggest the consideration of this "spoken-written" form as a particular form of its own. "Electronic discourse is a new variety of language that leads to significant variations in written structure of language and creates a kind of semi-speech that is between speaking and writing and it has its own features and graphology"[8]. It may seem insufficient to rely solely on binary opposition "spoken- written" which is still traditionally implemented in language studies. The "continuum" models proposed in the 80s (Chafe \&Tannen, 1987; Koch \& Oesterreicher, 1985) if applied to the description of digital writing and computer-mediated communication in general could be transformed into "triangular continuum model which has internet discourse as one of the extremes of the continuum" [9].

\section{Information about the authors:}

Vorotilina E.N. - Candidate of Philological Sciences, Associate Professor, Department of German and French Languages, Petrozavodsk State University (Petrozavodsk, Russia). E-mail: heldel@list.ru 
Dmitrieva V.A. - Senior Lecturer, Department of English, Petrozavodsk State University (Petrozavodsk, Russia).E-mail: valeria_67@mail.ru

\section{References}

1. McLuhan, M.: Understanding Media: The Extensions of Man (1964)

2. Crystal, D.: Language and the Internet. Cambridge: Cambridge University Press (2012)

3. Valiakhmetova, D.R.: Written colloquial speech in the context of the features of Internet discourse // Baudouin readings: Baudouin de Courtenay and modern linguistics: Intern. scientific conf. Kazan: Kazan Publishing House. Vol. 2, 7-9 (2001)

4. Amirova, T.A.: To the history and theory of graphemic. Moscow: Science, Main Edition of Oriental Literature (1977)

5. Schlobinski, P.: Grundfragen der Sprachwissenschaft. Eine Einführung in die Welt der Sprache(n). Göttingen: Uni-Taschenbücher (2014)

6.Eisenberg, P.: Das Fremdwort im Deutschen. Berlin: de Gruyter (2011)

7. Zehetmair, H.: Zukunft braucht Konservative. Freiburg im Breisgau: Verlag Herder (2009)

8. Abusa'Aleek, A.O.: Internet Linguistics: A Linguistic Analysis of Electronic Discourse as a New Variety of Language (2015)

9. Ketcham, E.: Internet discourse: the application of discourse analysis to instant messaging communication. Honors junior/senior projects. Paper 70 (2011)

Received 22 October 2019 